\title{
Un'esperienza di flipped classroom nella scuola media. Il teorema di Pitagora
}

\author{
A flipped classroom experience in lower \\ secondary school. The Pythagorean theorem
}

\author{
Alice Di Casola \\ Scuola media di Massagno, Svizzera
}

Sunto / In questo articolo è presentato un percorso didattico svolto in modalità flipped classroom in una scuola media. Tale modalità didattica, che può essere riassunta nell'espressione “teoria a casa e compiti a scuola”, può essere considerata innovativa in quanto introduce nuove tecnologie in aula, promuovendone un utilizzo consapevole. Il percorso, incentrato sul teorema di Pitagora, ha suscitato interesse negli studenti. Sono presentate tutte le lezioni svolte sull'argomento: l'attività introduttiva, la formulazione del teorema, le sue diverse dimostrazioni, l'implicazione inversa del teorema, esercizi vari e la verifica. Rispetto ad un'introduzione più tradizionale, si è riscontrato da parte degli allievi un gradimento maggiore e un apprendimento più radicato.

Parole chiave: flipped classroom; nuove tecnologie; scuola media; teorema di Pitagora.
Abstract / This article presents a didactic experience carried out in flipped classroom mode in a lower secondary school. This teaching method, which can be summarized as "theory at home and homework at school”, can be considered as innovative since it allows introducing new technologies in the classroom, promoting a conscious use of them. The experience, focused on the Pythagorean theorem, thrilled the students. The article reports on all the lessons on this theme: the introductory activity, the formulation of the theorem, its different proofs, the inverse implication of the theorem, various exercises, and the test. Compared to a more traditional introduction, the students were more motivated and their learning appeared to be more rooted.

Keywords: flipped classroom; new technologies; lower secondary school; Pythagorean theorem.

\section{Introduzione}

La flipped classroom, che tradotta letteralmente significa "classe capovolta", è un approccio sempre più sperimentato e utilizzato nei vari livelli scolastici. Si tratta di una metodologia didattica innovativa che presuppone un capovolgimento della classe o meglio dell'apprendimento con lo scopo di incrementarlo. Attraverso l'implementazione di questa modalità si vuole invertire la didattica classica, spostando l'acquisizione dei concetti a casa, rendendo tale fase più individuale, tramite diversi strumenti come testi, libri o video. In classe è invece prevista la rielaborazione dei concetti appresi tramite esercizi o situazioni problema in cui vi è la possibilità di mettere in pratica quanto imparato a casa. Uno dei vantaggi nell'implementare questa modalità didattica sta nella possibilità di riorganizzare i tempi e gli spazi d'apprendimento e creare degli ambienti di apprendimento che sostituiscano la classe "tradizionale" (Bergman \& Sams, 2016; Cecchinato \& Papa, 2016; Maglioni \& Biscaro, 2014).

In questo articolo si vuole presentare un percorso svolto in tale modalità didattica nella scuola media, esplicitando le varie attività realizzate e portando qualche spunto di riflessione per ulteriori miglioramenti. 
Per poter "capovolgere" la classe, risulta vincente l'utilizzo delle nuove tecnologie, per esempio per proporre la teoria sotto forma di video. La tecnologia è sempre più presente nella vita di tutti i giorni, anche dei giovanissimi, e il modo di imparare sta cambiando: basti pensare a come si può ottenere qualsiasi genere di informazione tramite l'uso di uno smartphone. Per questo motivo ci si chiede sempre di più cosa sia necessario insegnare a scuola e come. Accanto a questi cambiamenti e alla promozione da parte della scuola di una pedagogia attiva, vi sono pure le richieste sempre più esigenti da parte della società, che ricerca persone sempre più competenti le quali non solo possiedono conoscenze ma sanno anche organizzarle e utilizzarle in diverse situazioni anche complesse.

È proprio su questo terreno fertile che ha attecchito la flipped classroom (Sbaragli, Carotenuto \& Castelli, 2017). Essa, con la sua impostazione, si fonda su una pedagogia attiva grazie alla possibilità di lavorare in classe dopo uno studio a casa, concentrandosi anche e soprattutto sugli aspetti di collaborazione tra pari e condivisione delle proprie conoscenze.

Per valutare gli effetti di un tale approccio metodologico nell'insegnamento e apprendimento della matematica con gli studenti di scuola media, si è deciso di sperimentare un percorso in modalità flipped classroom, per poi confrontarlo con la didattica "tradizionale". ${ }^{1}$ L'argomento matematico proposto agli allievi di terza media in modalità flipped classroom è stato il teorema di Pitagora, che si presta molto bene alla creazione di video didattici, in quanto nella sua presentazione e dimostrazioni entra in gioco in modo forte il registro figurale.

Con I'approccio didattico della flipped classroom, viene enfatizzato il ruolo delle tecnologie in aula, sostenuto dal Piano di studio della scuola dell'obbligo ticinese (DECS, 2015), in cui si ritrova l'ambito di tecnologie e media tra i contesti di formazione generale. Inoltre, tale approccio permette lo sviluppo di competenze trasversali, quali ad esempio lo sviluppo personale (autoregolazione, consapevolezza di sé), la collaborazione (organizzazione del lavoro cooperativo, co-elaborazione), ma anche e soprattutto strategie d'apprendimento. Quest'ultima competenza è particolarmente messa in risalto in questo lavoro di ricerca in quanto gli allievi cercheranno di essere autonomi per quanto riguarda lo studio della teoria a casa e l'applicazione dei contenuti a lezione.

\section{Quadro teorico: la flipped classroom}

Come già anticipato, la flipped classroom è un approccio didattico che presuppone il capovolgimento della didattica "tradizionale", proponendo di fare a casa ciò che solitamente si fa a scuola, e a scuola ciò che solitamente si fa a casa. La pedagogia alla base della flipped classroom è la pedagogia attiva, che si trova in contrapposizione con la pedagogia trasmissiva, in cui il libro e il docente stanno al centro e l'apprendimento si realizza secondo uno schema classico che vede in sequenza: lezione frontale, studio individuale e verifica finale. La principale critica alla pedagogia trasmissiva è quella della passività degli alunni, che seguono la lezione condotta dal

1. Per una visione approfondita dei risultati della comparazione si rimanda al sito http://tesi.supsi.ch/2153/ 
docente senza possibilità di intervenire: «agli studenti è richiesto un ascolto attento e silente, e questo ruolo passivo non permette un apprendimento significativo in grado di provocare un cambiamento concettuale o lo svilupparsi di competenze» (Cecchinato \& Papa, 2016). Anche i ritmi in una lezione frontale sono determinati in modo univoco dal docente e spesso non tengono conto dei diversi tempi e stili di apprendimento degli allievi.

La flipped classroom nasce negli Stati Uniti tra la fine del secolo scorso e l'inizio del nuovo millennio, ma solo recentemente si riscontrano studi e pubblicazioni a riguardo. Oltre al rapporto FlisCo (Flipped classroom come approccio per lo sviluppo di competenze) sperimentato in Canton Ticino (Sbaragli, Carotenuto \& Castelli, 2017; Carotenuto \& Sbaragli, 2018), citiamo, tra le più conosciute ricerche, quelle di Bergmann e Sams (2012; 2016), due professori di chimica della Woodland Park High School in Colorado, la cui ricerca è stata un importante punto di riferimento per questo lavoro. Altre pubblicazioni sul tema sono state redatte da ricercatori dell'Università della Tasmania (Muir, 2017) e riguardano in particolare l'ambito matematico delle funzioni. Un'altra pubblicazione a cui questo lavoro fa riferimento è una ricerca italiana di Cecchinato e Papa (2016).

Per seguire tale approccio, va anche considerato che le nuove generazioni di studenti, chiamati "nativi digitali" in quanto cresciuti in ambienti in cui gli schermi interattivi sono molto diffusi, utilizzano internet come fonte primaria di ricerca, di acquisizione e di condivisione dei contenuti del sapere nonché per collaborare e cooperare tra pari attraverso strumenti quali Facebook, YouTube o altri social network (Ferri \& Moriggi, 2014). I nativi digitali sono dunque più abituati ad utilizzare tali mezzi di comunicazione e apprezzano maggiormente ricevere le informazioni in maniera veloce, preferendo solitamente immagini rispetto ad un testo e un video rispetto a una spiegazione scritta. Come già accennato in precedenza, anche le esigenze della società, e quindi della scuola, sono cambiate, perciò non ci si aspetta solamente che gli allievi possiedano conoscenze, ma che sappiano informarsi, interpretare criticamente tali informazioni e che sviluppino competenze da mobilitare in diversi contesti.

Ciò va collegato al ruolo del docente come "accompagnatore" piuttosto che come "saggio"; concetto che è stato introdotto da King nel 1993 in una sua pubblicazione in cui insiste sull'importanza di utilizzare il tempo in classe per approfondire gli argomenti piuttosto che per trasmettere le informazioni (Sbaragli, Carotenuto \& Castelli, 2017). Per questo motivo King è considerato uno dei pionieri della flipped classroom.

Un altro docente considerato tra i pionieri della flipped classroom è senz'altro Mazur (2007), professore di fisica ad Harvard, che propone un metodo per coinvolgere maggiormente gli studenti: prepararsi a casa sulla lezione successiva e poi discutere e approfondire in aula quanto imparato a casa.

Va anche ricordato Salman Amin Khan, che nel 2006 fonda la Khanacademy (https:// www.khanacademy.org), una piattaforma online contenente video lezioni di varie materie a cui si può accedere gratuitamente.

Ancor più recentemente, nel 2007 troviamo i docenti che spesso sono considerati tra gli ideatori della flipped classroom: Bergmann e Sams. I due insegnanti avevano iniziato a filmare le proprie lezioni per renderle disponibili agli allievi che spesso, e per vari motivi, erano assenti. In seguito sono passati a fornire in anticipo i video delle lezioni in modo che gli studenti potessero guardarli a casa e poi discuterne e approfondire i concetti in aula.

Da parte del gruppo di lavoro Flipped Learning Network (2014), gruppo di educa- 
tori che si è occupato della definizione del concetto di flipped classroom, sono stati redatti i cosiddetti "quattro pilastri" dell'apprendimento capovolto, i quali hanno I'acronimo FLIP: Flexible environment (ambiente flessibile), Learning culture (cultura dell'apprendimento), Intentional content (intenzionalità formativa), Professional educator (competenza professionale). È inoltre stata fornita una lista di indicatori per ognuno dei quattro pilastri, che ogni docente che si appresta a introdurre questa modalità didattica dovrebbe considerare e consultare per monitorare la propria attività.

Tabella 1

Quattro pilastri della flipped classroom (Sbaragli, Carotenuto \& Castelli, 2017).
- stabilire spazi e scansioni temporali che permettano agli studenti di interagire e riflettere sul loro apprendimento;

- osservare e monitorare costantemente gli studenti in modo da adattare e regolare la proposta formativa;

- fornire differenti opportunità agli studenti per apprendere i contenuti e dimostrare la loro padronanza.

- assicurare agli studenti occasioni per impegnarsi in attività significative che non prevedano la centralità dell'insegnante;

- supportare queste attività e renderle accessibili a tutti gli studenti attraverso la differenziazione e il feedback.

Intenzionalità $\quad$-focalizzarsi sui concetti chiave delle discipline di insegnamento facendo

formative $\quad$ in modo che gli studenti possano accedervi in autonomia;

- creare e/o selezionare contenuti significativi (tipicamente in forma audiovisiva) per i propri studenti;

- differenziare per rendere accessibili e rilevanti i contenuti proposti per tutti gli studenti.

Competenza

- fornire feedback individuali, di piccolo gruppo, di classe in tempo reale in base alle necessità;

- condurre valutazioni formative in itinere durante le lezioni attraverso l'osservazione e la documentazione di dati utili al prosieguo del lavoro formativo;

- collaborare e riflettere con gli altri docenti assumendosi la responsabilità del proprio sviluppo professionale.

\section{Metodologia}

La sperimentazione è stata proposta a una classe terza di corso attitudinale della scuola media di Massagno, formata da 15 allievi.

Il lavoro svolto può essere suddiviso nelle seguenti fasi: la progettazione e realizzazione del percorso didattico flipped classroom, e la creazione e somministrazione dei questionari volti a verificare l'impatto della sperimentazione sugli allievi. Per realizzare il percorso sono state previste anche le risorse necessarie per la sua implementazione e create le video lezioni da proporre agli studenti a casa.

L'argomento matematico scelto, il teorema di Pitagora, è centrale per la classe terza, ed è stato suddiviso in sette attività, diventate poi otto in seguito alla regolazione del percorso, che sono state proposte durante il periodo che va da fine gennaio a inizio marzo, con una frequenza di almeno un'attività a settimana. Durante questo periodo si sono limitati altri compiti che solitamente venivano assegnati settimanalmente a casa, per poter permettere agli allievi di concentrarsi sul percorso flipped 
classroom. Va tenuto conto che in Canton Ticino la scuola media è a tempo pieno. In questo articolo si riporta la descrizione del percorso didattico, per l'analisi dei risultati ottenuti dai questionari relativi alla percezione da parte degli studenti del percorso seguito in modalità flipped classroom si veda il lavoro di diploma al link http://tesi.supsi.ch/2153/.

\section{Descrizione dell'itinerario didattico}

\subsection{Risorse}

Durante il percorso si è scelto di utilizzare Google Classroom (https://classroom.google.com/) per lo scambio delle informazioni, dei video e delle consegne. Questa piattaforma si è rivelata molto adatta, in quanto facile e intuitiva da utilizzare, e già ben strutturata, ma allo stesso tempo con diverse impostazioni personalizzabili. Come possibile alternativa si era inizialmente pensato a Moodle, che però necessita di molto più tempo per personalizzare lo spazio online, nonché maggiori capacità tecniche. In Google Classroom, al momento dell'assegnazione di un compito è possibile allegare, oltre alla spiegazione, documenti, link e video. Anche la data, ed eventualmente l'ora della consegna del compito, può essere facilmente impostata al momento della creazione dello stesso, ed è possibile programmare il momento in cui un compito preparato precedentemente appare nella bacheca degli studenti. Inoltre, per assicurarsi che tutti gli allievi svolgano l'attività online, è possibile chiedere di segnalarlo in piattaforma, infatti ogni volta che viene assegnato un compito online, I'allievo ha la possibilità di "vistarlo" come completato. Se invece il compito deve essere consegnato digitalmente, gli studenti hanno la possibilità di creare il proprio documento e consegnarlo caricandolo in piattaforma. Inoltre, Google Classroom è molto adatto per un particolare tipo di compito, descritto nel dettaglio in seguito, che consiste nel poter assegnare un video diverso ad ogni gruppo senza che tutti possano vedere quello degli altri. È quindi possibile scegliere a quali studenti assegnare un determinato compito; aspetto fondamentale per poter differenziare le richieste.

Prima di iniziare a creare i video, che risulta dalla letteratura uno degli aspetti più gravosi dell'implementazione della flipped classroom, si è iniziato a guardare cosa fosse già presente in rete su questo argomento. Sono stati reperiti su YouTube sei video che potevano essere adatti alle esigenze del percorso: tre del canale Schooltoonchannel - di cui due sono stati uniti - e tre che riguardavano le dimostrazioni. A questi video sono stati fatti solo alcuni tagli e alcune inquadrature con il programma iMovie (presente su tutti i dispositivi Apple). Altri video sulle dimostrazioni sono stati rintracciati sul portale della Zanichelli. A questi, con lo stesso programma, è stato tolto I'audio, inserita una musica ed effettuato qualche taglio. Ė stato poi registrato con un nuovo audio il video sulla classificazione dei triangoli che era già stato utilizzato e creato all'interno del progetto FlisCo. ${ }^{2}$

Per alcune attività è stato preparato un quiz in Socrative, http://www.socrative.com/, un sito che permette di creare vari tipi di domande e soprattutto di avere le rispo-

2. Tutti i video utilizzati e prodotti nel corso di questa esperienza didattica sono disponibili su richiesta scrivendo a dfa.rivistaddm@supsi.ch 
ste istantaneamente, man mano che gli allievi rispondono, ognuno con le proprie tempistiche. In Socrative è possibile creare quiz con domande a scelta multipla, vero/ falso o con brevi risposte aperte, potendo inserire anche immagini nel testo della domanda e segnare la risposta corretta con un'eventuale spiegazione, se si desidera che essa venga visualizzata dagli studenti, al termine del quiz. Il quiz è stato sottoposto durante due attività come primo approccio in classe dopo aver studiato la teoria a casa. Si tratta perciò di un possibile momento in cui si riprende quanto svolto a casa. Per fare in modo che ogni studente possa fornire la propria risposta è dunque necessario trovarsi in un'aula di informatica oppure, come in questo caso, fare in modo che i ragazzi abbiano a disposizione i loro cellulari per poter rispondere alle domande, ognuno dal proprio dispositivo. Non avendo il wifi a disposizione, è stato comunque possibile connettersi, in quanto chi aveva internet a disposizione lo ha condiviso con alcuni compagni.

\subsection{Il percorso didattico}

Si riporta di seguito una tabella riassuntiva del percorso sperimentato in cui vengono evidenziate le attività a casa e quelle in aula e dove si mostrano i momenti in cui sono state raccolte le informazioni tramite i questionari per poter rispondere alle più ampie domande di ricerca del lavoro di diploma.

\begin{tabular}{|c|c|c|c|}
\hline Numero attività & A casa & A scuola & Durata \\
\hline 1. Preconoscenze & $\begin{array}{l}\text { Raccolta e verifica delle } \\
\text { preconoscenze sulla } \\
\text { classificazione dei triangoli } \\
\text { (Video } 1,1.52 \mathrm{~min} \text { ) }\end{array}$ & & \\
\hline \multicolumn{4}{|c|}{ Questionario 1 dopo l'attività a casa } \\
\hline & & $\begin{array}{l}\text { Quiz con Socrative su } \\
\text { quanto visto a casa } \\
\text { (Allegato 1) }\end{array}$ & $1 \mathrm{~h}$ \\
\hline \multicolumn{4}{|c|}{ Questionario 1 dopo l'attività a scuola } \\
\hline 2. II teorema & $\begin{array}{l}\text { Video tratto da Schooltoon } \\
\text { (Video 2, } 3.06 \mathrm{~min} \text { ) }\end{array}$ & & \\
\hline \multicolumn{4}{|c|}{ Questionario 2 dopo l'attività a casa } \\
\hline & & $\begin{array}{l}\text { Quiz con Socrative } \\
\text { (Allegato 2) e messa a } \\
\text { punto dell'enunciato del } \\
\text { teorema. Primi esercizi } \\
\text { (Allegato 3) e riflessione su } \\
\text { che cos'è una } \\
\text { dimostrazione }\end{array}$ & $2 \mathrm{~h}$ \\
\hline \multicolumn{4}{|c|}{ Questionario 2 dopo l'attività a scuola } \\
\hline 3. Dimostrazioni & $\begin{array}{l}\text { Assegnazione di cinque } \\
\text { diverse dimostrazioni a } \\
\text { cinque gruppi di tre allievi } \\
\text { ciascuno. Il compito } \\
\text { consiste nel registrare un } \\
\text { audio che spieghi la } \\
\text { dimostrazione del video } \\
\text { assegnato }\end{array}$ & $\begin{array}{l}\text { Presentazione delle } \\
\text { dimostrazioni ai compagni } \\
\text { e riflessione su quanto } \\
\text { visto. Risoluzione di } \\
\text { esercizi. }\end{array}$ & $2 \mathrm{~h}$ \\
\hline
\end{tabular}


Tabella 2

Percorso flipped classroom.

\begin{tabular}{|c|c|c|c|}
\hline $\begin{array}{l}\text { 4. Storia del teorema e } \\
\text { sua implicazione } \\
\text { inversa }\end{array}$ & $\begin{array}{l}\text { Quiz per iscritto sul } \\
\text { contenuto del video tratto } \\
\text { da Schooltoon sulla parte } \\
\text { storica (Allegato 4) } \\
\text { (Video 3, } 8.39 \mathrm{~min} \text { ) }\end{array}$ & & \\
\hline \multicolumn{4}{|c|}{ Questionario 3 dopo l'attività a casa } \\
\hline & & $\begin{array}{l}\text { Correzione del quiz, } \\
\text { inverso del teorema } \\
\text { (Allegato 5), esercizi }\end{array}$ & $1 \mathrm{~h}$ \\
\hline \multicolumn{4}{|c|}{ Questionario 3 dopo l'attività a scuola } \\
\hline $\begin{array}{l}\text { 5. Il teorema di Pitagora } \\
\text { nella realtà }\end{array}$ & $\begin{array}{l}\text { Pensare a quali situazioni } \\
\text { della vita reale si può appli- } \\
\text { care il teorema di Pitagora }\end{array}$ & $\begin{array}{l}\text { Creazione di problemi più } \\
\text { complessi a partire da } \\
\text { quello che hanno pensato } \\
\text { a casa (Allegato 6) }\end{array}$ & $1 \mathrm{~h}$ \\
\hline $\begin{array}{l}\text { 6. Situazione problema } \\
\text { finale }\end{array}$ & & $\begin{array}{l}\text { Situazione problema sul } \\
\text { bang sonico } \\
\text { (Allegato 7) }\end{array}$ & $1 \mathrm{~h}$ \\
\hline 7. Esercitazione & & Risoluzione di esercizi & $1 \mathrm{~h}$ \\
\hline \multicolumn{4}{|c|}{ Questionario di fine percorso } \\
\hline 8. Verifica & & $\begin{array}{l}\text { Verifica monotematica } \\
\text { (Allegato 8) }\end{array}$ & $2 \mathrm{~h}$ \\
\hline
\end{tabular}

Come si può notare dalla tabella, non tutte le attività sono state svolte in modalità flipped classroom, ma si è cercato di adattare la modalità a seconda delle esigenze. In questa sperimentazione è risultato importante aggiungere in corso d'opera una lezione di esercitazione in vista della verifica finale.

Si riporta di seguito una descrizione più puntuale del percorso.

\section{Attività 1 a casa - Prerequisiti.}

La prima attività verteva su un ripasso del tema dei triangoli e della relativa classificazione, argomento che viene già affrontato alla scuola elementare e che abbiamo ripreso concentrandoci sugli elementi e sulle caratteristiche del triangolo rettangolo che non tutti avevano affrontato nel livello scolastico precedente.

A casa agli alunni è stato chiesto di visionare un video in cui venivano ripresi questi concetti di base per affrontare il teorema di Pitagora. Nella Figura 1, è riportato come la docente poteva visualizzare il compito assegnato in piattaforma agli studenti.

\section{E 103 Alice Di Casol}

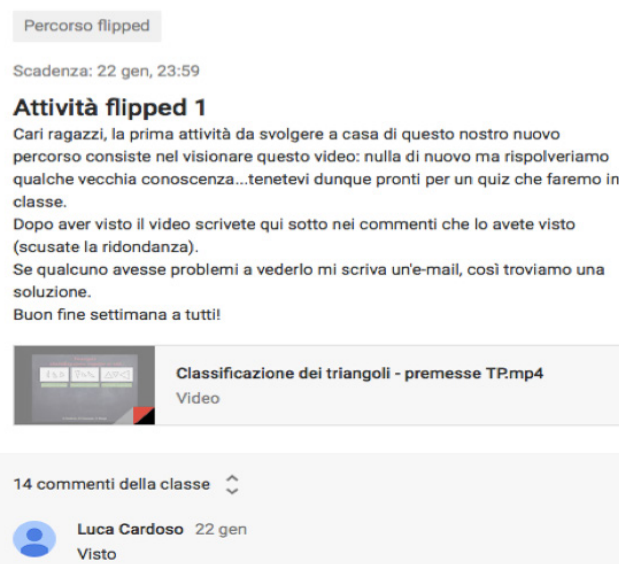


Attività 1 a scuola- Prerequisiti.

In aula l'attività consisteva nel sottoporre un quiz tramite Socrative, pensato per testare i prerequisiti degli allievi riguardo al tema ripassato (Allegato 1). Le domande erano del tipo: "esiste un triangolo rettangolo isoscele?" oppure "esiste un triangolo rettangolo equilatero?". Lo scopo era indagare se, viste le diverse caratteristiche dei triangoli, gli allievi fossero in grado di metterle in relazione per stabilire l'esistenza di determinati triangoli esplicitando le motivazioni sottostanti le diverse scelte. Nella Figura 2, viene mostrato un esempio di come un allievo poteva visualizzare la domanda.

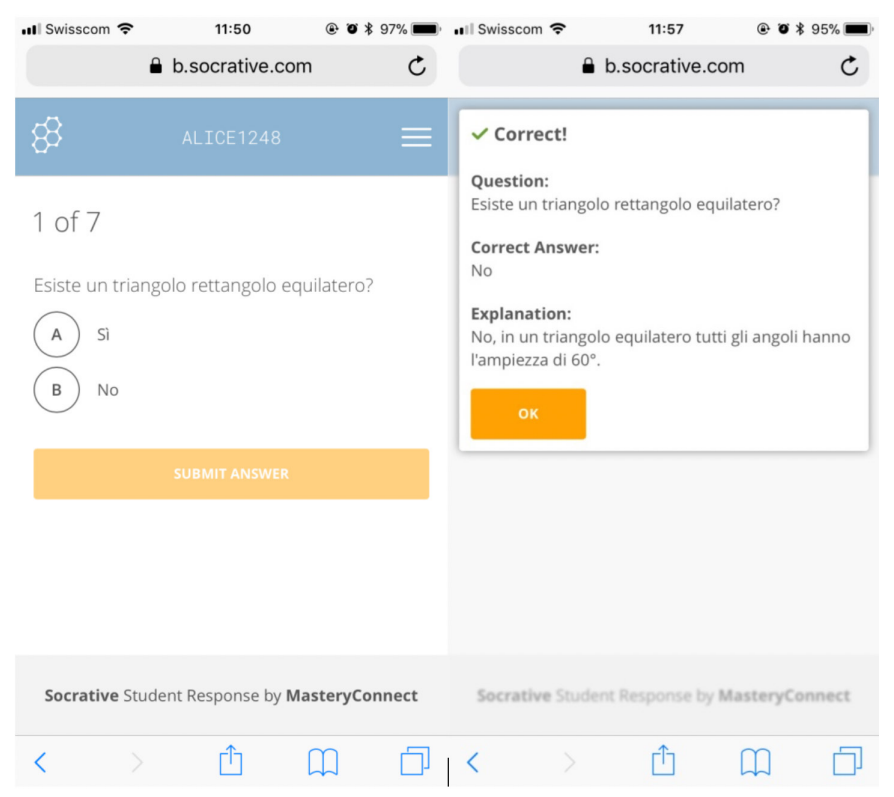

Un esempio di domanda per l'allievo in Socrative (sinistra) e la risposta

fornita con relativa spiegazione (destra).

Figura 3

Proiezione alla lavagna dei risultati del quiz in tempo reale.

Nel frattempo, simultaneamente, venivano proiettati i risultati alla lavagna, come si può vedere in Figura 3

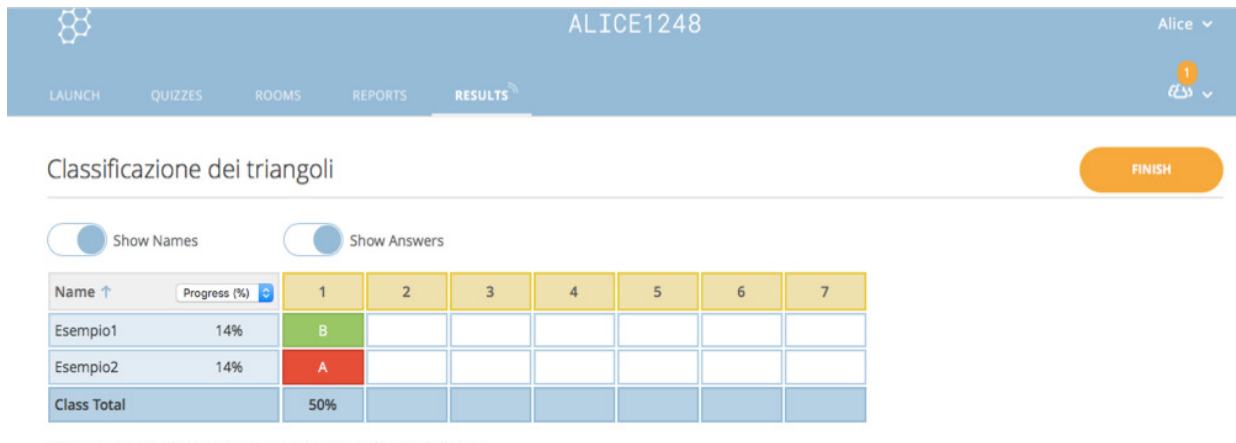

Essendo la prima volta che gli studenti utilizzavano Socrative, è servito un po' di tempo per far sì che tutti gli allievi fossero connessi alla rete e collegati correttamente al quiz. Dopo aver risposto al quiz, è seguita una correzione comune con una discussione su quanto emerso. La lezione si è conclusa svelando che l'argomento delle lezioni successive avrebbe riguardato i triangoli rettangoli. 
Riguardo a questa prima attività si può affermare che, pur essendo risultata per gli studenti piuttosto semplice e per alcuni necessaria solo come bilancio, è risultata un buon punto di partenza per avviare il percorso.

Attività 2 a casa - II teorema.

Anche in questa lezione la richiesta a casa è stata di visionare un video animato tratto da Schooltoon (Figura 4) contenente la spiegazione del teorema e di tenersi pronti per i dubbi da sciogliere e le applicazioni in aula.

Figura 4

I personaggi del video animato.

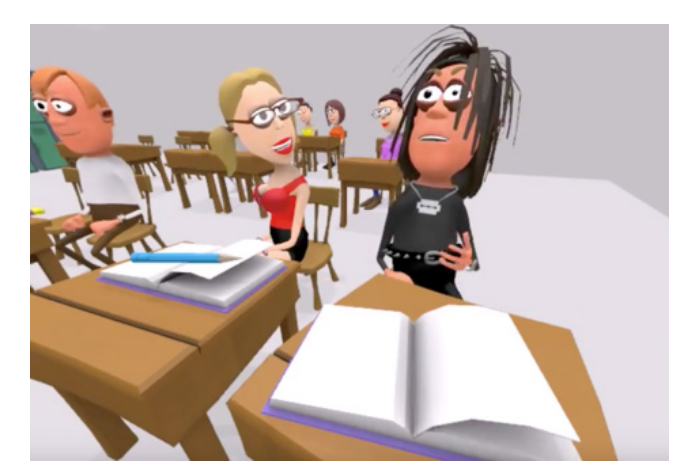

Assistere a una lezione in questa modalità può essere accattivante per gli studenti per vari motivi: non è il loro insegnante a parlare e possono guardarlo al proprio ritmo (eventualmente tornando indietro e ricominciando) in contesti diversi. II video inizia con un professore "nonnetto" (così viene chiamato in Schooltoon) che presenta il teorema come qualcosa di fondamentale della geometria euclidea. Dopo aver ripreso il concetto di triangolo rettangolo, il professore elenca i nomi dei suoi lati. In seguito, esprime l'enunciato del teorema come segue "in un triangolo rettangolo l'area del quadrato costruito sull'ipotenusa è sempre equivalente alla somma delle aree dei quadrati costruiti sui cateti" (Figura 5).

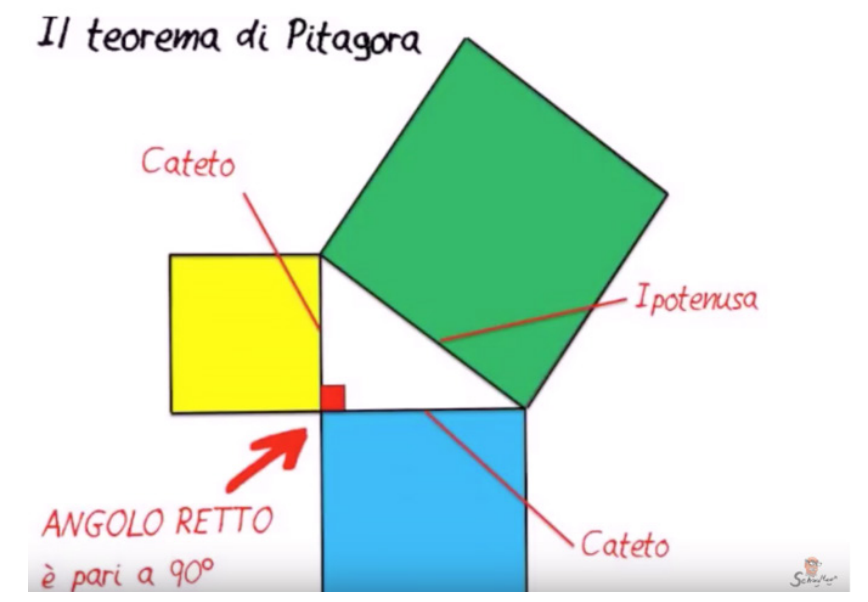

Il professore fa poi un accenno all'esistenza di molteplici dimostrazioni, mostrando un'animazione in cui tramite lo spostamento di un liquido si cerca di convincere della veridicità del teorema. 
Figura 6

Il professore mostra alla lavagna alcune dimostrazioni, una delle quali è un'animazione.

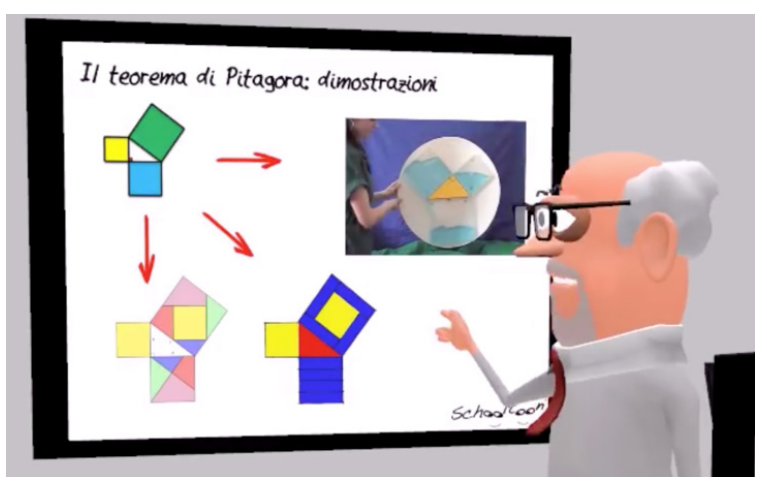

In seguito nel video un allievo chiede quale sia I'utilità di questo teorema nella vita reale, e così viene messo l'accento sul fatto che nelle applicazioni del teorema non si costruiranno fisicamente dei quadrati sui lati di triangoli rettangoli, bensì esso può permette di calcolare la lunghezza di uno dei lati conoscendo la lunghezza degli altri due. In seguito, il professore esprime e spiega il teorema nella forma algebrica equivalente: $a^{2}+b^{2}=c^{2}$.

Si è ritenuto questo video adatto per gli studenti in quanto corretto dal punto di vista matematico, con un accenno alle dimostrazioni e in cui è già presente l'importanza dell'applicazione.

Attività 2 a scuola - II teorema.

A lezione si sono proposte, sempre tramite Socrative, cinque domande (Allegato 2) collegate ai contenuti proposti nel video da visionare a casa per compito. La prima domanda, che riguarda le ipotesi del teorema, mostrava un'immagine con alcuni triangoli e si chiedeva in quali di essi è possibile applicare il teorema. La seconda e la terza domanda (Figure $7-8$ ) vertevano invece sulla nomenclatura del triangolo rettangolo essenziale per la comprensione dell'enunciato. La quarta domanda riguardava l'applicazione del teorema.

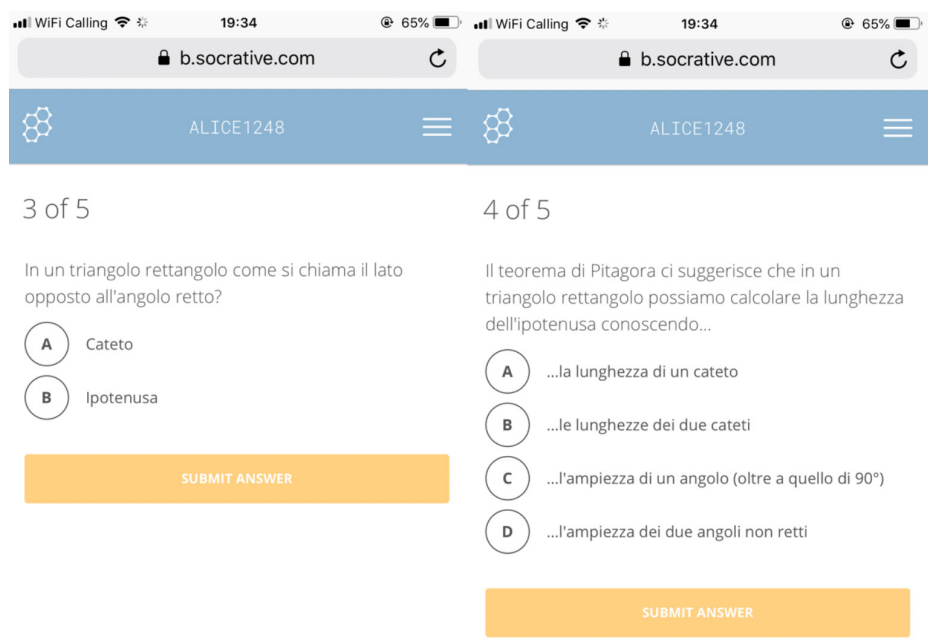


Figura 8

Esempi di domande su Socrative.

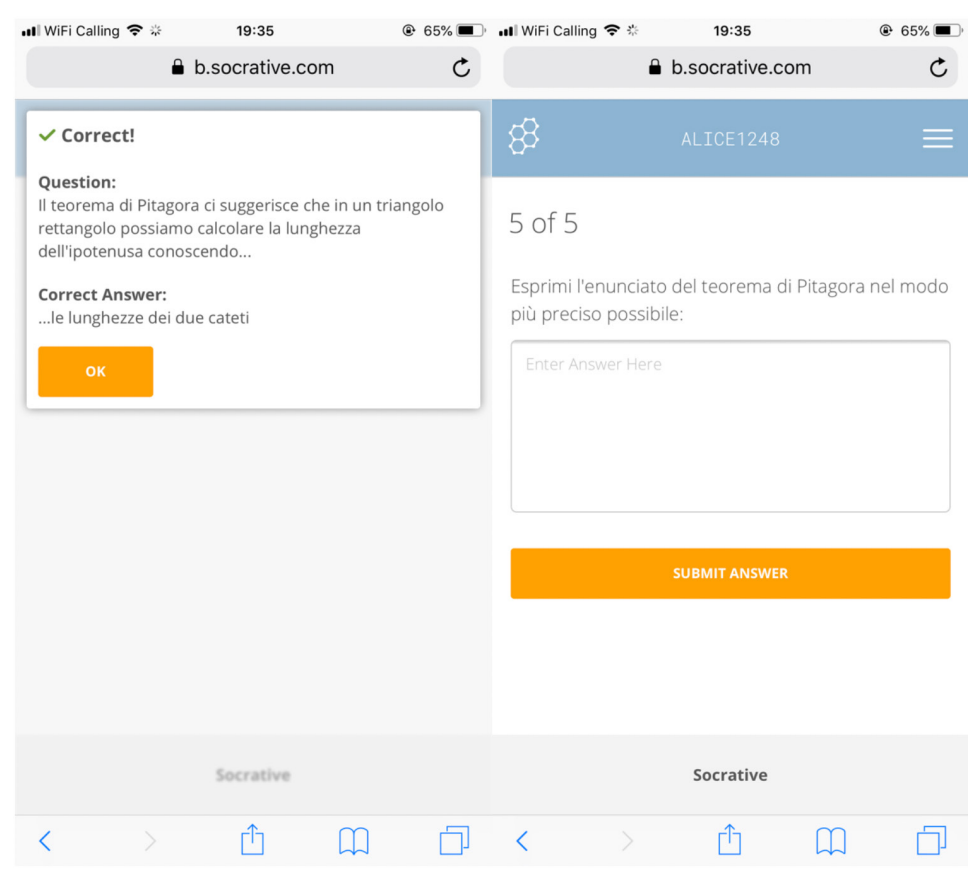

Infine l'ultima domanda, che a differenza delle altre era aperta e non a scelta multipla, chiedeva di formulare nel modo più preciso possibile l'enunciato del teorema. Durante la correzione, avvenuta subito dopo il quiz, questa domanda, proposta volutamente per ultima, ha avviato la discussione sull'enunciato del teorema, in particolare su quanto fosse importante essere precisi nel linguaggio utilizzato, sulle ipotesi e sulla tesi. Questa lezione è risultata molto efficace, grazie in particolare al video veramente adatto alle necessità del percorso. Nella seconda parte della lezione sono stati proposti alcuni esercizi per permettere agli studenti di mettere in campo ciò che avevano appreso (Allegato 3). Per partire da richieste più semplici, i primi due esercizi riguardavano esclusivamente triangoli rettangoli, dunque chiedevano di calcolare la lunghezza di un lato conoscendo le lunghezze degli altri due, e in un caso utilizzare poi la lunghezza del lato trovato per calcolare un perimetro. In un altro esercizio si chiedeva di calcolare il perimetro e l'area di un rettangolo, conoscendo la lunghezza di una diagonale e di un suo lato. In questo modo gli studenti potevano riflettere sul fatto che il teorema di Pitagora può essere applicato ogni volta che viene individuato un triangolo rettangolo all'interno di una figura data. Un ultimo esercizio, un po' più legato alla realtà, chiedeva di calcolare la lunghezza di una scala appoggiata al muro. Come di consueto, si sono proposti altri esercizi tratti dal libro di testo per chi finiva prima il compito. La maggior parte degli studenti ha potuto finire gli esercizi, gli altri li hanno terminati a casa. Prima di concludere la lezione, tornando ai concetti di enunciato, tesi e ipotesi, si è affrontato il tema della dimostrazione, per far comprendere di cosa si tratta e introdurre la lezione successiva.

\section{Attività 3 a casa - Dimostrazioni.}

Per questa attività gli alunni sono stati suddivisi in cinque gruppi omogenei ai quali è stato assegnato un video senza audio contenente una dimostrazione del teorema, richiedendo ad ogni gruppo di creare un audio che potesse accompagnare il video, spiegando la dimostrazione. Per questa attività, le dimostrazioni scelte sono state, ad eccezione di una, tutte geometriche. Si è ritenuto infatti più semplice per gli allievi 
Figura 9

Dimostrazione con

quadretti (sinistra)

Figura 10

Dimostrazione di Perigal (destra). descrivere un'immagine rispetto a spiegare una scrittura algebrica. I compiti assegnati erano di difficoltà differenti e sono stati attribuiti in base alle competenze degli allievi. La dimostrazione ritenuta più semplice è quella in cui si suddividono i quadrati costruiti su cateto minore e maggiore rispettivamente in 9 e 16 quadratini (come indicato in Figura 9) che poi vengono riposizionati a formare il quadrato costruito sull' $i$ potenusa, mostrando così che quest'ultimo ha la stessa estensione dei due quadrati costruiti sui cateti. A un altro gruppo è stata consegnata la dimostrazione di Perigal (Figura 10) e ad altri due gruppi le dimostrazioni tratte dal sito della Zanichelli (Figura 11 -12), le quali si basano sulla stessa scomposizione di figure, ma una coinvolge più I'aspetto geometrico, mentre l'altra più l'aspetto algebrico.
Figura 11

Dimostrazione geometrica tratta da Zanichelli.
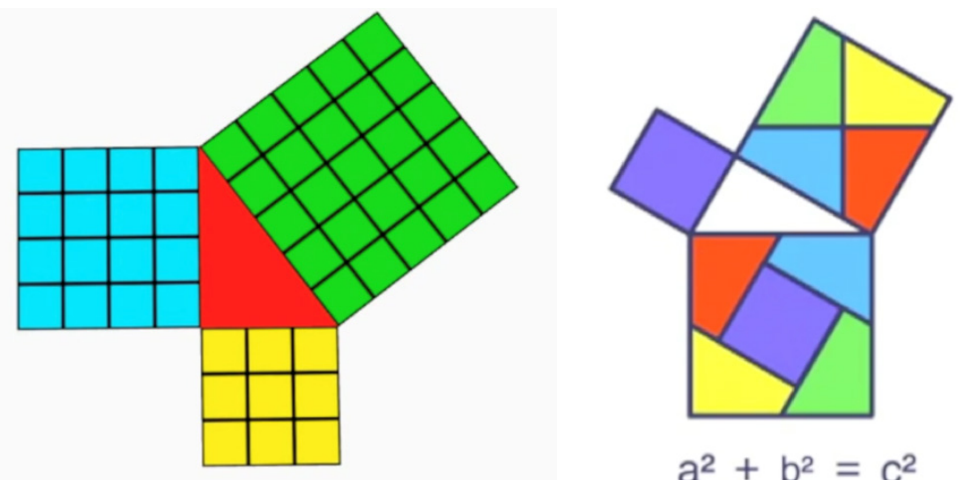

$a^{2}+b^{2}=c^{2}$

$$
a^{2}+b^{2}=c^{2}
$$
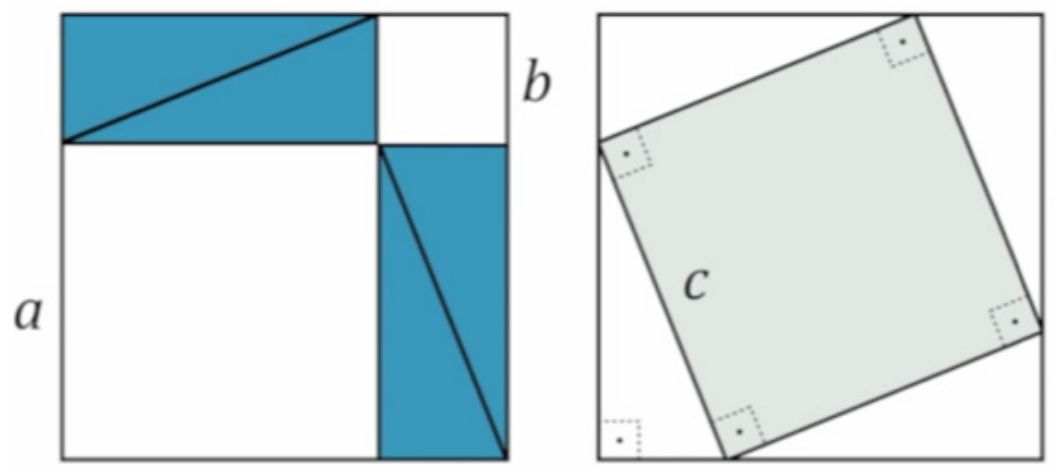

\section{Teorema di Pitagora}

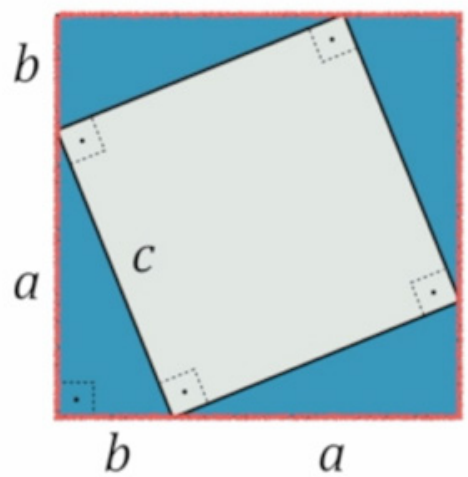

$$
a^{2}+2 a b+b^{2}=c^{2}+2 a b
$$

$$
a^{2}+b^{2}=c^{2}
$$


La dimostrazione più complicata da commentare, essendo il video più veloce come sequenza di immagini, è una dimostrazione chiamata "cinese", mostrata in Figura 13. Gli studenti avevano pochissimo tempo a disposizione (1:49 $\mathrm{min}$ ) per spiegare ciò che accadeva nel video.

Figura 13

Dimostrazione cinese.

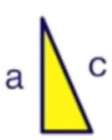

b
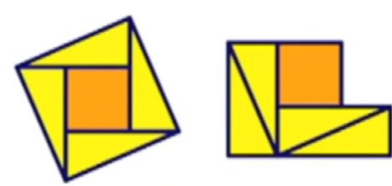
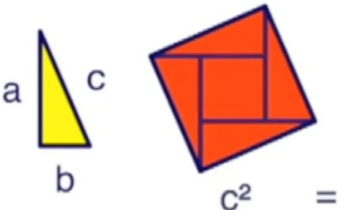

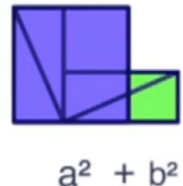

Trattandosi di un compito diverso da quelli consegnati in precedenza dal docente si è deciso di mostrare un esempio in classe di come si poteva commentare un video, e sono stati dati alcuni punti da seguire per preparare l'audio richiesto: capire la dimostrazione, scrivere un testo che potesse essere idoneo e provare a leggerlo mentre scorrevano le sequenze del video. Solo dopo aver rispettato queste istruzioni il gruppo poteva passare alla registrazione dell'audio e consegnare il proprio compito (traccia audio) in piattaforma.

Attività 3 a scuola - Dimostrazioni.

In aula ogni gruppo ha presentato ai compagni i video completi di spiegazione e si è chiesto agli studenti di fare dei commenti al lavoro svolto dai compagni. Per guidarli in questo compito, prima di iniziare con la proiezione, si sono decisi assieme alcuni criteri che si sarebbero considerati, quali la correttezza del linguaggio italiano, matematico e correttezza di senso. Non si trattava di un compito semplice perché i video non erano stati visionati preventivamente dagli altri gruppi che li avrebbero dovuti comprendere in quel momento grazie alla spiegazione dei compagni. È stato un momento di condivisione efficace e il risultato ottenuto dagli studenti è stato molto positivo. È anche stato interessante per gli studenti avere delle critiche da parte dei compagni, anche se spesso erano gli stessi autori ad accorgersi di alcuni errori commessi. Nel tempo rimanente della lezione si sono affrontati ancora esercizi del libro, riguardanti semplici applicazioni del teorema.

Attività 4 a casa - Storia del teorema e sua implicazione inversa.

A casa, agli allievi è stato chiesto di guardare un video, sempre tratto dal canale YouTube Schooltoon, dove venivano mostrate alcune applicazioni del teorema di Pitagora nella realtà e qualche cenno sulla storia del teorema. Per accertarsi che i concetti del video fossero stati assimilati, si è pensato di proporre un quiz cartaceo (Allegato 4) da svolgere a casa durante la visione.

Attività 4 a scuola - Storia del teorema e sua implicazione inversa.

In classe si è corretto il quiz e si è affrontato l'inverso del teorema, in modalità classica, non capovolta. La lezione è iniziata in modalità dialogata, ricordando quanto visto sul teorema e riprendendo la struttura logica di un teorema (se... allora...). Con I'aiuto di una scheda (Allegato 5) è stato chiesto agli allievi di costruire alcuni triangoli date le lunghezze dei lati e di classificarli: nello specifico, si trattava di un triangolo acutangolo scaleno e un triangolo rettangolo scaleno. Dopo aver mostrato a tutta la classe la costruzione di tali triangoli con GeoGebra, si sono costruiti i quadrati sopra i lati $a, b$ e $c$, e si è così notato che nel caso del triangolo rettangolo valeva l'uguaglianza $a^{2}+b^{2}=c^{2}$. Dopo tale osservazione si è quindi espresso l'inverso del teorema, 
mettendolo a confronto con il teorema già noto, e si è mostrata una dimostrazione. Infine, sono stati proposti degli esercizi su quanto appena appreso. In un esercizio, date le lunghezze dei tre lati si chiedeva se fosse possibile costruire un triangolo e se esso fosse rettangolo. In un altro, data una figura composta da triangoli, si chiedeva quali di essi fossero rettangoli (Figura 14). Su questo esercizio si era discusso a lungo in quanto, avendo a disposizione solo le lunghezze di due lati di un triangolo, non era possibile determinare se si trattasse di un triangolo rettangolo (stiamo parlando del triangolo $A B F)$.

2. Data la figura a lato, dove $A B C, A C E$ e $A B F$ sono tre triangoli (con $A B C$ rettangolo). Determinare se ACB e ABF sono dei triangoli rettangoli.

L'ultimo esercizio, sempre legato all'inverso del teorema, era invece di natura più algebrica.

Se si dovesse riproporre il percorso in futuro, la parte a casa di questa attività sarebbe impostata in modo diverso, cioè senza proporre un quiz cartaceo. Lo si potrebbe proporre per esempio inserendo le domande durante la visione del video, oppure semplicemente proponendo un questionario online, così da poter eventualmente raccogliere le risposte degli allievi e riprendere in classe solo il necessario invece di correggere tutto il quiz.

Attività 5 a casa - II teorema di Pitagora nella realtà.

In questa lezione è stato chiesto ai ragazzi di pensare, e in seguito scrivere, un problema della realtà in cui fosse necessario applicare il teorema di Pitagora. Ad esempio, qualche studente ha pensato di applicarlo al tetto di una casa.

Attività 5 a scuola - II teorema di Pitagora nella realtà.

In classe, a partire da quanto elaborato a casa, gli studenti suddivisi in gruppi dovevano scegliere un problema tra quelli elaborati dai componenti del proprio gruppo, eventualmente completarlo, per poi consegnarlo ai compagni di altri gruppi allo scopo di risolverlo (Allegato 6).

Le proposte elaborate a casa non sono risultate particolarmente interessanti, per questo si sono dovuti guidare un po' gli studenti partendo dalle loro idee e convinzioni. Ad esempio, in un gruppo uno studente aveva pensato al tetto di una casa, e mantenendo quell'idea si è riusciti a formulare un problema riguardante la stima della quantità di tegole necessarie per costruirlo. Per gli studenti è risultato molto motivante sapere che i compagni avrebbero dovuto risolvere il loro problema. Tutti i 
gruppi cercavano quindi di complicarlo, fornendo meno dati possibili. Per esempio, un gruppo ha avuto l'idea di lavorare sulle dimensioni in pollici dei televisori, e hanno poi deciso di togliere un dato aggiungendo però l'informazione sul rapporto tra le lunghezze.

Attività 6 a scuola - Situazione problema finale.

Al termine di questo percorso si è pensato di proporre un problema (Figura 15) in cui la chiave di risoluzione era appunto il teorema di Pitagora (Allegato 7). La situazione, tratta da un evento realmente accaduto in Ticino, chiedeva di scoprire la posizione di un aereo F/A-18 al momento del superamento del muro del suono, conoscendo il momento in cui in due località si era sentito il botto, chiamato bang o boom sonico.

\section{Due $F / A-18$ fanno tremare il Sopraceneri}
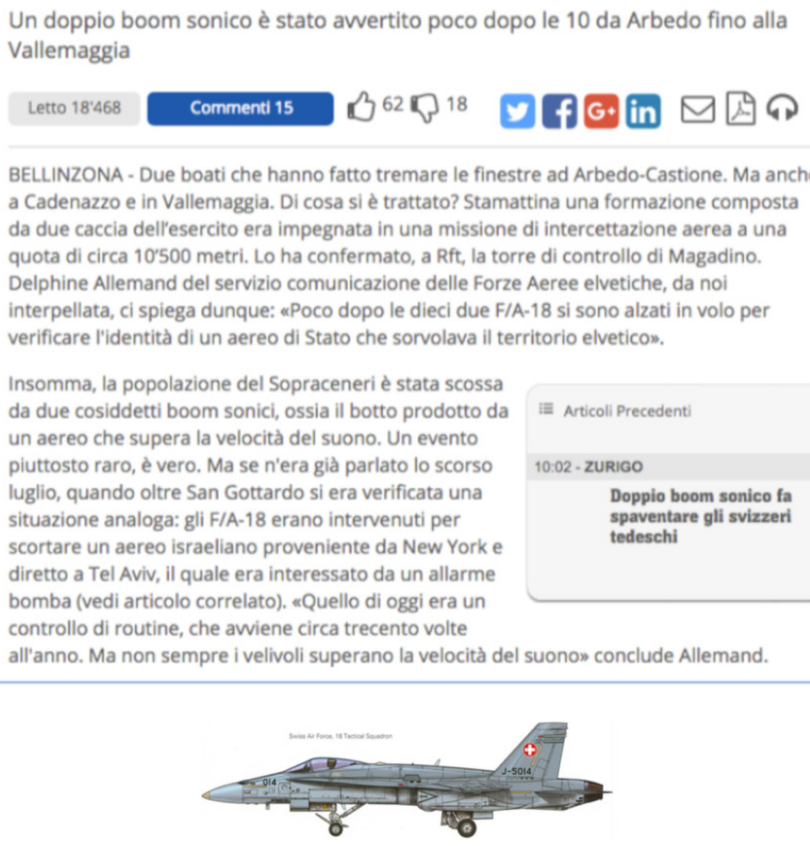

Le informazioni presenti nella consegna erano la quota degli F/A-18 al momento del botto, le altitudini delle due città e i secondi che sono passati prima che in ognuna delle due si sentisse il suono. Nella prima parte della scheda era riportato l'articolo pubblicato online in cui si descriveva l'accaduto e nella seconda era spiegato il fenomeno fisico del bang sonico. Gli studenti dovevano quindi calcolare la distanza percorsa dal suono e tramite il teorema di Pitagora conoscere la distanza in linea d'aria tra ognuna delle due città e la posizione degli aerei. Con questo dato e l'aiuto di una cartina, gli allievi sono riusciti a scoprire due possibili posizioni degli F/A-18 per poi escluderne una in quanto si trovava in Italia (nell'articolo era specificato che l'avvenimento era accaduto in Svizzera).

Questa situazione ha motivato molto gli studenti, perché si trattava di un problema reale e di primo impatto difficile da risolvere. Nonostante ciò, gli allievi si sono dimostrati tutti in grado di risolvere il problema senza nessun particolare aiuto. Infatti, una volta rappresentata la situazione, si sono subito accorti di come avrebbero potuto applicare il teorema di Pitagora. 
Attività 7 a scuola - Esercitazione.

Questa attività è stata inserita perché ci si è resi conto della necessità di svolgere altri esercizi prima di affrontare la verifica finale. In particolare in questa lezione sono stati risolti i problemi creati dai compagni durante l'attività 5 e sono stati svolti vari altri esercizi tratti dal libro. Gli studenti non hanno incontrato particolari difficoltà: infatti l'argomento era stato ben compreso e si trattava solamente di esercitarsi. Gli esercizi proposti chiedevano per esempio di calcolare il perimetro di poligoni rappresentati sul piano cartesiano oppure di calcolare area e perimetro di figure geometriche particolari grazie all'applicazione del teorema.

Attività 8 a scuola - Verifica finale.

Al termine del percorso gli allievi hanno affrontato una verifica sul teorema di Pitagora (Allegato 8).

La verifica è stata una di quelle meglio riuscite dell'anno scolastico e non è stata riscontrata nessuna particolare difficoltà. L'esercizio meno riuscito, come era prevedibile, è stato il secondo, relativo ad una rampa, in cui era necessario ragionare sull'area del triangolo per riuscire a scoprire le misure mancanti (Figura 16).

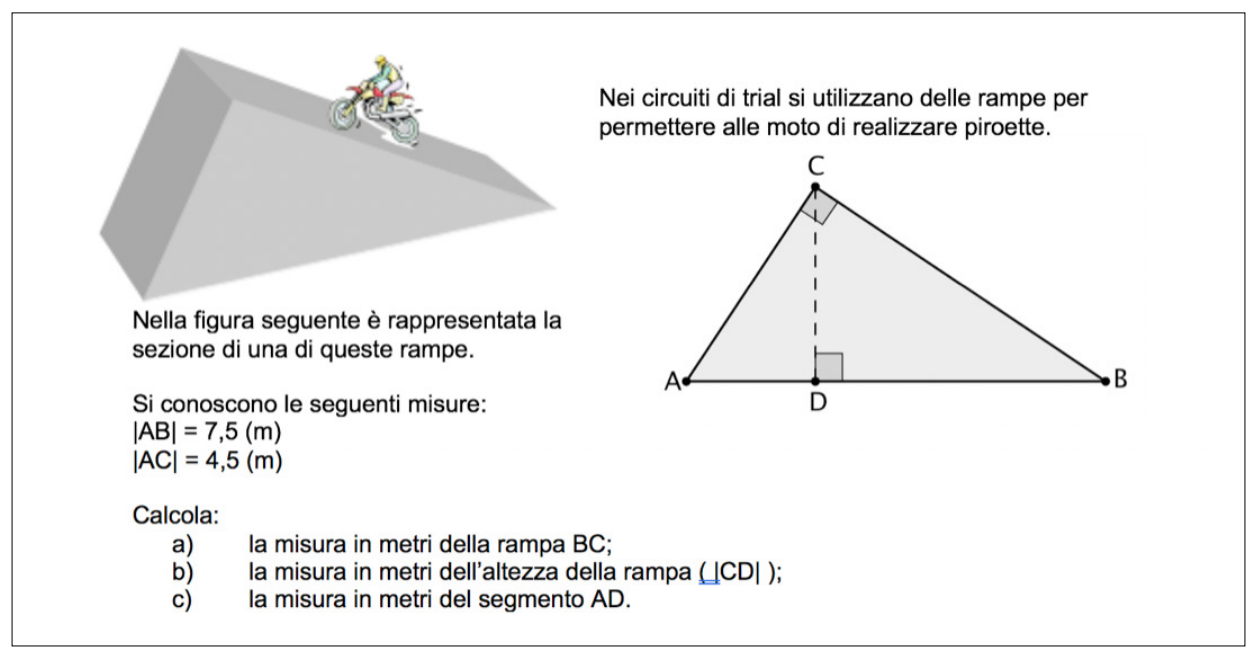

\section{Problematiche e punti di forza del percorso}

\subsection{Sintesi dei risultati}

Dai questionari è generalmente emerso che gli studenti hanno apprezzato i video, trovandoli chiari ed esaustivi. Analizzando separatamente i risultati dei questionari dopo ogni singola attività, è emerso che la prima è stata percepita come semplice, chiara, e non portava a distrazioni. La seconda attività è stata ritenuta molto interessante e utile, e solo la terza, probabilmente a causa della lunghezza del video, è stata ritenuta più impegnativa e in alcuni casi alcuni allievi avrebbero preferito avere una spiegazione da parte della docente. Inoltre, questa video lezione è stata la più rivista da diversi studenti, anche se ritenuta adeguata: gli allievi hanno ritenuto che il contenuto fosse diverso dai precedenti e richiedesse maggior attenzione anche se, matematicamente parlando, non vi era nulla di nuovo.

A fine percorso, praticamente tutti gli studenti hanno confermato l'efficacia di ogni 
Figura 17

Distribuzione delle risposte alla seconda domanda del questionario finale. attività svolta: dalla visione del video, alle spiegazioni e attività in aula, ai compiti a casa (Figura 17).

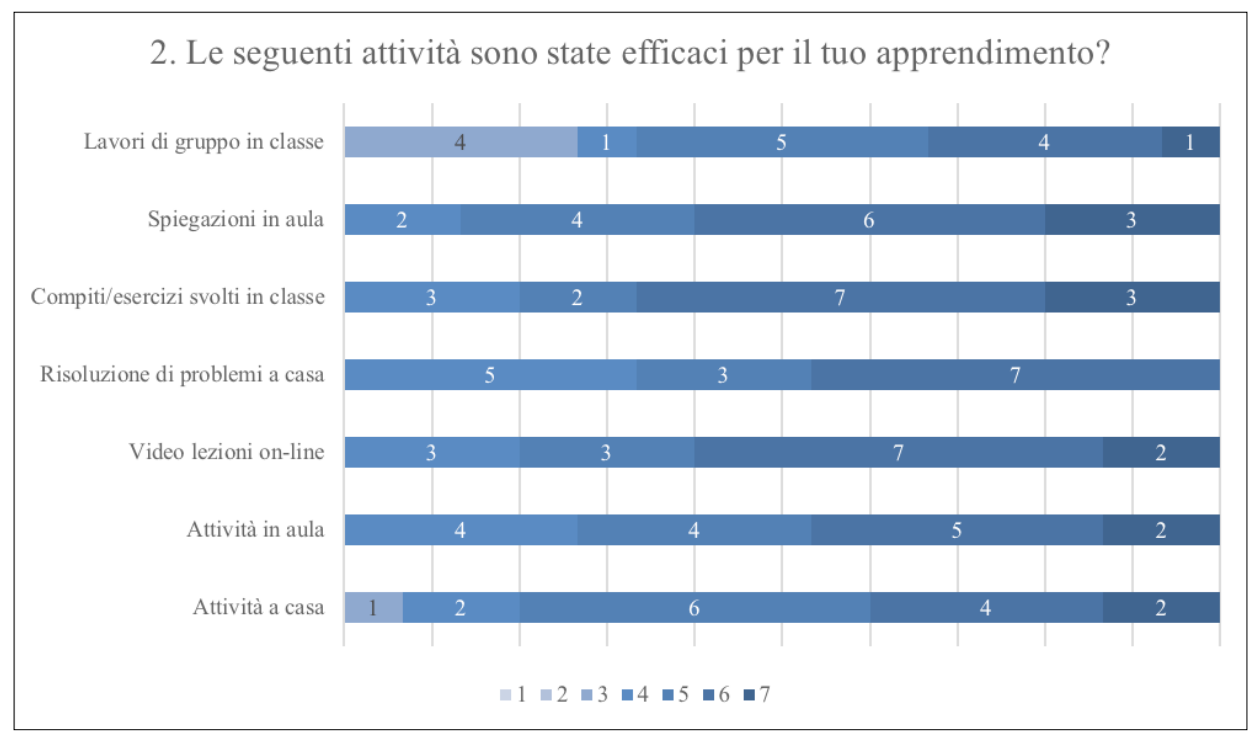

Anche nel confronto tra i due approcci (Figura 18), quello tradizionale e quello sperimentale, applicato su temi diversi, la flipped classroom è emersa come migliore in tutti i casi tranne due. Sono stati presi in considerazione i seguenti aspetti: uso efficace del tempo, acquisizione di concetti matematici, qualità delle interazioni con la docente e con i compagni, coinvolgimento personale, efficacia del percorso e gradevolezza. Come si può immaginare, i due aspetti risultati migliori nella modalità tradizionale, sono quelli della qualità delle interazioni con la docente e i compagni. Si noti che la gradevolezza è l'aspetto che presenta uno scarto maggiore tra le due metodologie.

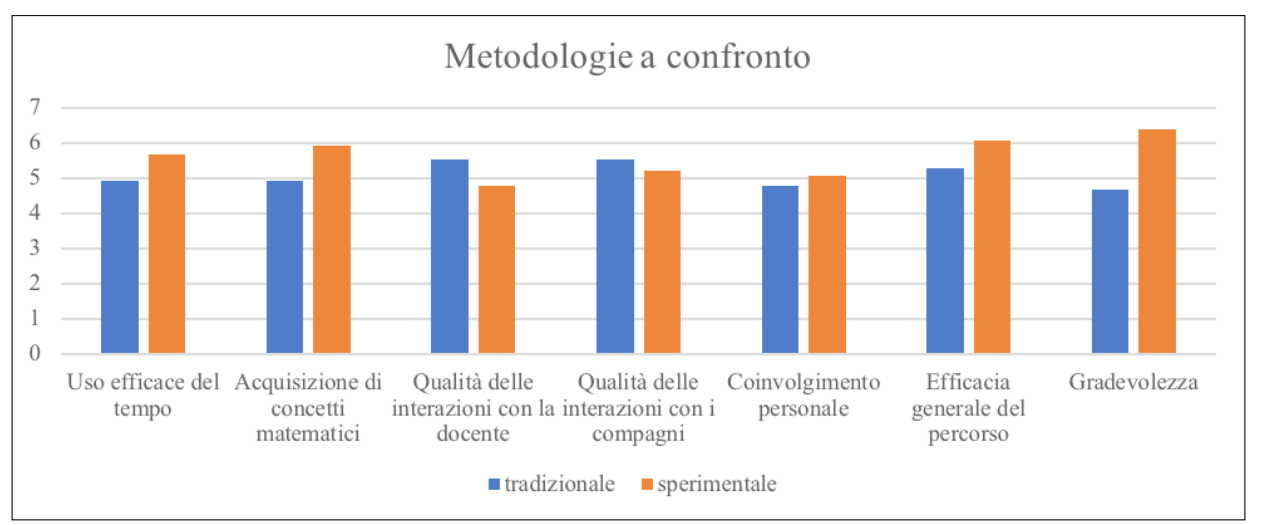

Dalle ultime domande aperte, in cui si chiedevano gli aspetti migliori e peggiori di queste lezioni "sperimentali", è emerso che i migliori sono: poter riguardare il video e quindi prendersi il proprio tempo oppure non perderlo inutilmente, aver imparato meglio e in maniera più efficace e divertente, avere una maggior comunicazione perché si è sempre in contatto e l'utilizzo delle nuove tecnologie. In particolare, uno studente ha espresso il desiderio di affrontare altri argomenti in questa modalità. 
Figura 19

Aspetti migliori della metodologia sperimentata, allieva 1.
Si mostrano di seguito le risposte di tre allieve che ben riassumono quanto emerso (Figure 19 - 22).

In clasee non si perdeva troppo tempo con le spiegationi e si agiva di pic. Poter uscforre di appareochi ételtronici per la scuola stimda di più.

Si pú connunicare per eventoali domande e chiarimenti

in qualstasi momento.

Secondo me è stato bello fore le lezioni sperimentali perché:

- quando guordovemo i video potevemo, in caso di dubbi, riguerdorli mentre in clesse nan passiomo fore il "repley" delle spiegezone.

- A scude potevamo porre domende sul lavoro suolto e caso.

- Mi é precuuto poter sfruttore le nuove tecnologie.

- Trav comodo poter fare i compiti con il computer.

- é intenesscate poler fore metemetica e cose

Lavorare a casa guardando i video e potendo comunizare con la docente in qualsia momento è molto coinvolgente e divertente, invoglia adi imparare e quindi.è efficace e utile.

A volte le spiegazioni si capiscono meglio "dal vivo", ma comunque non sono mancate quindi non vedo aspetti negativi.

Queste ultime due risposte hanno dunque ancor più messo in evidenza l'apprezzamento che gli studenti hanno provato per questo percorso.

\subsection{Il punto di vista didattico-disciplinare}

Come confermato dal questionario finale, dalle opinioni degli studenti a fine percorso, e soprattutto dalla positiva riuscita della verifica finale, si ritiene che il percorso abbia avuto successo a livello didattico-disciplinare. Come già accennato, non si ritiene che questo approccio debba essere utilizzato per affrontare ogni argomento, ma sicuramente si pone come valida alternativa. Come si è potuto sperimentare in questo percorso, specialmente per problemi in cui la visualizzazione è importante, la visione di un video può aiutare la comprensione di un argomento.

Anche per quanto riguarda l'apprendimento, si può affermare che il bilancio è stato positivo. È infatti chiaro per tutti che per apprendere ognuno di noi ha bisogno dei propri tempi, e la flipped classroom si adatta bene a questa caratteristica dell'apprendimento, permettendo ad ogni studente di prendersi i propri tempi per apprendere. 


\subsection{Metodologia e strumenti utilizzati}

Progettare e realizzare un percorso didattico in questa modalità, dovendo partire da zero, richiede molto lavoro. Paragonata alla progettazione di un percorso svolto in modalità tradizionale, la preparazione richiede più tempo in quanto inizialmente non si è abituati a progettare in maniera capovolta. Inoltre, la realizzazione delle video lezioni può risultare complessa, ma è anche un'attività stimolante per il docente che si trova ad analizzare il tema da un punto di vista diverso, riflettendo sugli elementi essenziali per una spiegazione breve ed efficace. Si ritiene quindi che, con un po' di pratica, questa modalità didattica possa essere una valida alternativa alle lezioni più tradizionali. Si sottolinea anche il fatto che il docente, come accaduto in questo percorso, si può ispirare a materiale e risorse disponibili in rete, selezionandoli e adattandoli in base alle proprie esigenze.

Come sottolineato nella descrizione delle attività, vi sono solo alcuni aspetti da modificare in futuro, come per esempio la somministrazione online del quiz dopo o durante il video sulla storia del teorema. Si può pertanto affermare che il percorso così come è stato strutturato risulta vincente per i docenti, per esempio per la possibilità offerta dalla piattaforma di differenziare un compito a casa. Inoltre si tratta di un nuovo modo di lavorare che può essere alternato alla didattica tradizionale. Insegnando in questo modo, è anche possibile sviluppare diverse competenze trasversali: sviluppare I'uso della lingua e delle tecnologie, la capacità di organizzare il lavoro a casa, presentare il proprio lavoro ai compagni, collaborare e molto altro ancora. Dal punto di vista degli studenti, essi si sono sempre mostrati motivati ad affrontare le varie attività a casa e a scuola erano contenti di poter essere più attivi del solito. È stato sicuramente interessante e nuovo per loro dover svolgere un compito come quello delle dimostrazioni, creando un audio di un video muto, per spiegare a parole ciò che era intuibile dalla visione. Poter essere affiancati durante i momenti di esercitazione e affrontare invece a casa ciò che altrimenti verrebbe presentato frontalmente, risulta sicuramente vincente per l'apprendimento. Dal punto di vista del docente va anche ricordata come molto positiva la possibilità offerta dalla piattaforma di differenziare il compito degli studenti a casa.

\section{6 condusion}

Nonostante le ricerche su questo argomento riguardino soprattutto scuole superiori e il livello terziario, è interessante notare come sia stato possibile proporre la modalità capovolta anche con studenti più giovani, non ancora così autonomi nello studio. Se guidati, anch'essi sono in grado di approfittare degli aspetti positivi di quest'approccio, come ad esempio potersi prendere il proprio tempo per capire i nuovi argomenti. Nel progettare un percorso flipped classroom è però importante - specialmente con studenti così giovani - tenere conto della complessità dell'argomento e considerare se esso non è forse più adatto per essere introdotto con una didattica tradizionale. Anche i video non dovrebbero superare una certa lunghezza ed essere adatti, cioè comprensibili per chi li utilizzerà. A tal proposito si ritiene che sia importante creare personalmente i materiali (nello specifico, i video) o valutare, selezionare e riadattare materiali esistenti, in modo che rispecchino esattamente le necessità delle lezioni. È praticamente impossibile trovare qualcosa di adatto sotto tutti gli aspetti. 
Questo percorso, pur essendo proposto ad un'unica classe di soli 15 allievi, ha portato un ulteriore esempio di come la metodologia flipped classroom possa essere percepita efficace ed essere apprezzata da parte degli studenti. Inoltre, essendo la tecnologia sempre più presente nella vita di tutti i giorni, è fondamentale poterla introdurre nelle nostre scuole per poter aiutare i ragazzi ad averne un uso consapevole, aspetto questo, che riguarda il contesto di formazione generale tecnologie e media da sviluppare secondo le indicazioni del Piano di Studio (DECS, 2015).

La modalità didattica qui sperimentata è quindi una delle possibilità per rendere la matematica più apprezzata dagli studenti, perché tiene conto dei differenti ritmi di apprendimento: permette cioè una differenziazione, elemento essenziale per la riuscita di tutti.

\section{Bibliografia}

Bergmann, A., \& Sams, J. (2012). Flip your Classroom: Reach every Student in every Class every Day. New York: Intl Society for Tecnology.

Bergmann, A., \& Sams, J. (2016). Flip your Classroom. Firenze: Giunti.

Carotenuto, G., \& Sbaragli, S. (2018). Flipped classroom per la formazione insegnanti: una ricerca sulla percezione degli studenti. Didattica della matematica. Dalla ricerca alle pratiche d'aula, 3, 7-34. DOI: 10.33683/ddm.18.3.1

Cecchinato, G., \& Papa, R. (2016). Flipped Classroom: un nuovo modo di insegnare e apprendere. Torino: UTET.

DECS, Divisione della Scuola. (2015). Piano di studio della scuola dell'obbligo ticinese. Bellinzona.

Ferri, P., \& Moriggi, S. (2014). La classe di Bayes. Note metodologiche, epistemologiche ed operative per una reale digitalizzazione della didattica nella scuola italiana. Journal of Educational, Cultural and Psychological Studies(10), 135-151.

Khan, S. A. (2006). Tratto da Khan Academy: https://www.khanacademy.org

King, A. (1993). From sage on the stage to guide on the side. College teaching, 41(1), 30-35.

Maglioni, M., \& Biscaro, F. (2014). La classe capovolta. Trento: Erickson.

Mazur, E. (1997). Peer Instruction: A User's Manual. Upper Saddle RIver: Prentice Hall.

Muir, T. (2017). The enactment of a flipped classroom approach in senior secondary mathematics class and its impact on student engagement. Proceedings of the 41 st conference of the International Group for the Psychology of Mathematics Education, 3, 281-288.

Network, F. L. (2014). The Four Pillars of F-L-I-P. Tratto da Flip Learning: https://flippedlearning. org/wp-content/uploads/2016/07/FLIP handout FNL Web.pdf

Sbaragli, S., Carotenuto, G., \& Castelli, L. (2017). Flipped classroom come approccio per lo sviluppo di competenze. Rapporto interdipardimentale dell'Asse 8, SUPSI.

\section{Autore/Alice Di Casola}

Scuola media di Massagno - Svizzera

alice.dicasola@edu.ti.ch 